\title{
Design of an add-on device for transform a standard wheelchair on an affordable and motorized
}

\author{
Benedikt Prusas $^{\text {ab* }^{*}, K \text { Karim Mansoor }}{ }^{\mathrm{b}}$, L. Engelhardt ${ }^{\mathrm{b}}$, Saku Pirtiläb ${ }^{\mathrm{b}}$ Bas Walgers $^{\mathrm{b}}$, \\ Louisa Lukoschek ${ }^{b}$, Beatriz Defez García ${ }^{c}$, Ismael Lengua ${ }^{c}$ and Guillermo Peris- \\ Fajarnés $^{\mathrm{c}^{*}}$ \\ ${ }^{a}$ Hochschule Düsseldorf, Germany, e-mail: *Benedikt.Prusas@study.hs-duesseldorf.de \\ ${ }^{\mathrm{b}}$ EUROPEAN PROJECT SEMESTER, ETSID-Universitat Politècnica de València, Valencia, Spain \\ ${ }^{\mathrm{c} C I T G-U n i v e r s i t a t ~ P o l i t e ̀ c n i c a ~ d e ~ V a l e ̀ n c i a, ~ V a l e n c i a, ~ S p a i n, ~ " ~ e-m a i l: ~ g p e r i s @ u p v . e s ~}$
}

\begin{abstract}
In the following article a design to adapt a low-cost power unit to a conventional wheelchair is presented. The adaptation is carried out looking for a simple, low cost solution that does not involve any modification or structural alteration of the wheelchair itself. In this work we present a proposal of a mechanical adaptation that will allow connecting a self-balancing scooter to a wheelchair and be controlled by the user as well as by another person. So that the pushing effort is eliminated.
\end{abstract}

Keywords: motorized wheelchair, electric powered, self-balancing scooter, mobility

\section{Introduction}

The world population is growing, and the demographic transition is in place, increasing the number of elderly people (United Nations Department of Economic and Social Affairs, 2017). Thus, the need for wheelchairs are increasing by approximately 3500 units per day ("Analysis of Wheelchair Need", 2019). Given that the quality of life is directly correlated to the mobility of a person, (Hudakova \& Hornakova, 2011) making mobility accessible is an important topic. The world report on ageing and health states: "When assistive devices are available, affordable and appropriate to older people's needs and their environments, their mobility, independence and participation can be greatly enhanced." (WHO, 2015). Progress and efforts have been made in this regard. Such as of the wheelchair foundation 
which distributed over a million wheelchairs ("Wheelchair Foundation", 2019) or innovative devices such as the all-terrain wheelchair, developed by Amos Winter (Winter, 2012). Also, attempts have been made to make power assisted mobility solutions more accessible as well. For example, students of the Bingham Young University have founded the Open Wheelchair Foundation and published instructions to build an electric wheelchair (Hollingshead, 2015).

This paper is a contribution to explore options to make power assisted mobility more accessible for those whose physical condition is not sufficient enough to operate a manual wheelchair and to discuss in detail, whether there is a way to make motorized wheelchair solutions more affordable. This work has been created as a university project with restrictions such as a four-month time frame and a limited budget. Based on those limitations the decision was made, to benefit from mass production of already existing consumer electronics. Chosen was a self-balancing scooter. The requirements for this design included factors such as that it could be used daily and amongst other things, to be able to switch it on and off from the seated position as well as being able to push the wheelchair manually.

\section{Design}

For this design, with the purpose to motorize a wheelchair, the following general conditions had to be met:

- Use of a self-balancing scooter as a way of propulsion.

- Universal design allowing connection to different wheelchair-models, with no modification to the wheelchair itself.

- Solutions that can be made without big machinery.

- $\quad$ Plug \& play system which the user can attach without any help.

- Controlling the self-balancing scooter without the use of feet.

- Being able to remove the self-balancing scooter and comfortably transport the construction while traveling.

- Stopping when releasing the controls. 
The self-balancing scooter, as seen in Figure 1, has a gyroscope system which can balance a person standing on it. You are able to control the scooter by shifting your weight forth or back, which gets registered by pressure pads, located on the top of the standing platform. If you rotate the pedal backwards the wheel will also revolve to the same direction. It is mandatory to have pressure on the pedals in order to control it. Both of the wheels are able to spin independently from each other, this allows a very tight turning radius. (Patent CN204223088U, 2015)

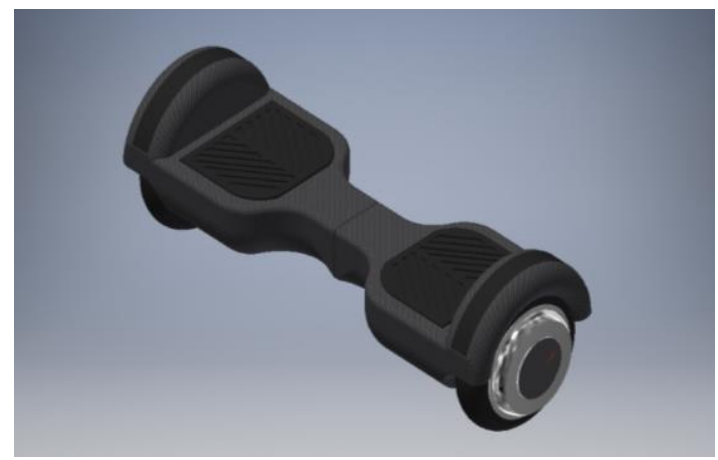

Fig. 1: A self-balancing scooter

The idea of a self-balancing scooter behind a wheelchair is like the 'aidwheels system'. With the aidwheel system, there is a link between the self-balancing scooter and the wheelchair so that the wheelchair can be pushed by the self-balancing scooter ("AidWheels Mobility Solutions", 2019). This approach has the restriction that an extra person needs to stand on the self-balancing scooter to control it.

In order to realize all the above-mentioned requirements, the developed design is based on placing the self-balancing scooter as an electric drive behind the main wheels of the wheelchair, without making any structural modification of the original wheelchair in order to be used and adapted to as many models as possible. The main wheels are lifted from the ground allowing a smaller turning radius to be used independently from the wheelchair model.

The total design consists of two mounting plates, one middle axis, steering assist, two steering wheels, and a support frame. The middle axis is connected to the mounting plates in a way that allows rotation. The steering assist has the purpose to return the control pedals back to a neutral position when the steering is untouched. Movements of the steering are transmitted to the control pedals through a tensioned cable. 
In order to connect the scooter to the frame and the rest of the design, a welded and bent construction made from metal was built according to the measurements of the selfbalancing scooter. This construction, as shown in Figure 2, now functions as a fixing point for the rest of the construction. Springs are used to move the control pedals back to their initial position, where no movement will result. This will be referred to as the steering assist.

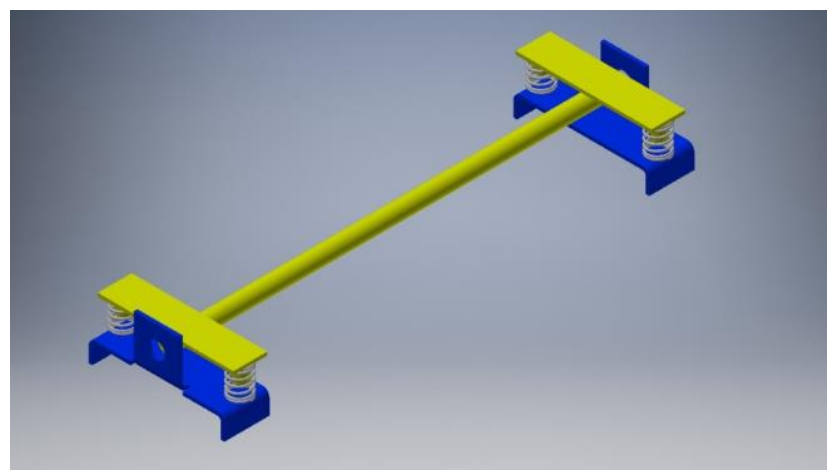

Fig 2: Mounting Plate with Steering Assist

The supporting frame has the purpose to lift the main wheels of the wheelchair up from the ground. This allows usage with different kinds of wheels and wheelchair models through the redirecting of the weight of the person to the self-balancing scooter. Traction of the scooter wheels is granted, and pressure can then be exerted on the pressure-pads of the selfbalancing scooter, which is required to control it. The disadvantage of lifting the main wheels is that it causes a change in the sitting angle. At this point, a trade-off has to be made, lifting the wheels up higher allowing the wheels to overcome more obstacles but makes the seat angle closer to a horizontal position, which could lead to the driver falling out of the wheelchair. The decision was made in favour of the safety of the user by only lifting the wheels a few centimetres from the ground.

The idea is to design a very light and easy working construction to lift up the main wheels and get the connection. Iron is cheap and robust, but aluminium should be the final material and it is perfectly suitable for this task. For the initial prototype, four wooden beams get screwed together to a construction in which the wheels of the wheelchairs fit perfectly. The newly formed wooden construction is now placed under the wheels.

In the rear part, it is connected with the steel steering assist, which is described earlier in this article. The weight of the wheelchair is now transferred to the scooter and its wheels. 


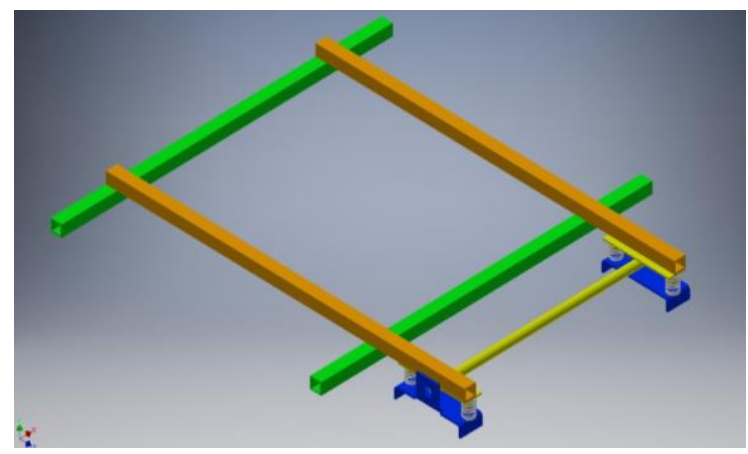

Fig 3: Assembled construction

In the front part, the wooden construction is tied to the wheelchair on each side, lifting up the mainwheels. The distance between the wheels and the ground can be adjusted by the length of the ropes.

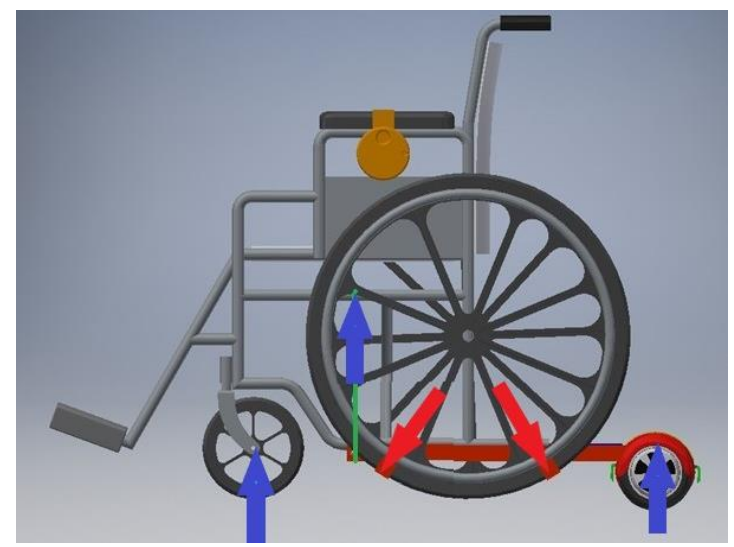

Fig 4: Force vectors

The wheelchair is sitting with its main wheels on the wooden frame, which causes the red forces. The red forces lead to reaction forces, marked as blue arrows, in the ropes (green) as well as in the self-balanced scooter and front wheels. 




Fig 5: Assembled Construction with Wheelchair

In order to control the self-balancing scooter normally the person using it would change the center of gravity from their feet back and forth on the pressure pads to control the wheels individually. This allows for steering by making the wheels spin in the opposite direction from each other. Excluding the use of feet, the controlling has to be done with both hands. Each wheel can individually be controlled into going forwards or backwards allowing for movement, steering and breaking. To control each wheel individually a system with tension cables is used which is most similar to that of a bicycle shifting system.

When rotating the handlebar to shift into a new gear a rotating force is being translated into a pulling force. This is used to tilt the self-balancing scooter backwards or forward. A clamp is used to connect the control to each side of the wheelchair on any desired location as shown on fig. 6 .

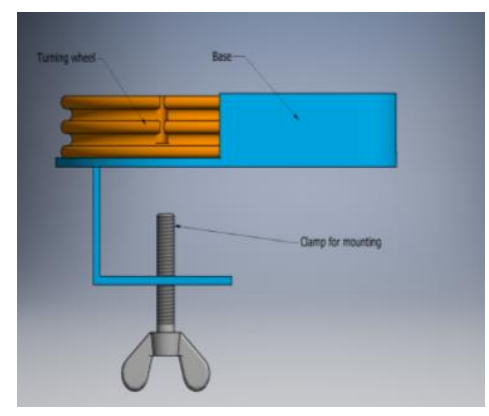

Fig 6: Controlling System 
Each control unit has two tension cables in it. These cables are the same ones used on bikes. On the tip of the cable, there is a metal block.

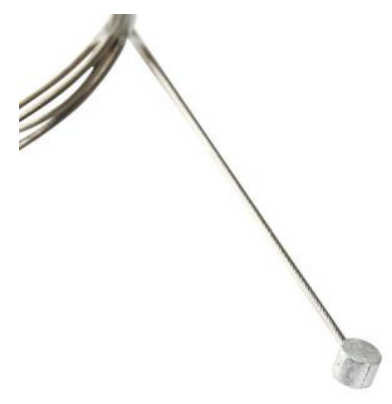

Fig 7: Cable for controlling System

This block slides into the turning wheel (shown on fig. 8)

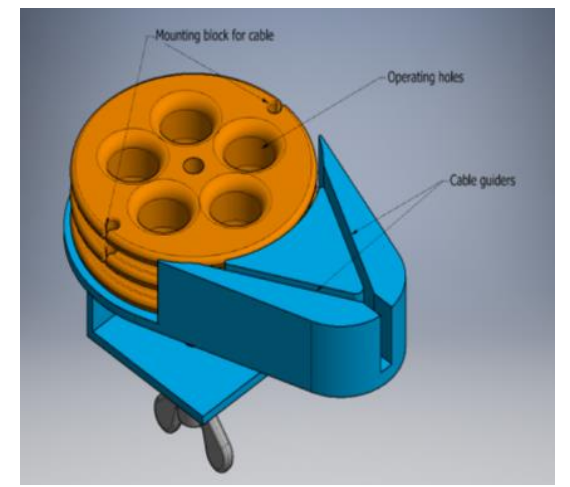

Fig 8: Opened Case with cable channels



Fig 9: locked case 
When operating the wheel one of the cables will be pulled and the other one will be loose, allowing the balancing board to tilt to the front or to the back.

If there is the need for a second person to steer the wheelchair, the additional person can stand on the steering system, which is displayed in figure 2. By shifting their weight as if they would ride the self-balancing scooter without the extra construction, the wheelchair can be steered externally.

A foldable solution is also being considered. When the electric drive is not needed, the construction can be easily folded together and does not consume much space. Therefore, the whole construction is much easier to transport and can even be carried around in the back of a wheelchair. The difficulty is that the construction and especially the beams must be still strong enough to carry the weight of a heavy person. For this purpose, a different construction for the self-balancing scooter has been provided, which allows the construction to be folded up.

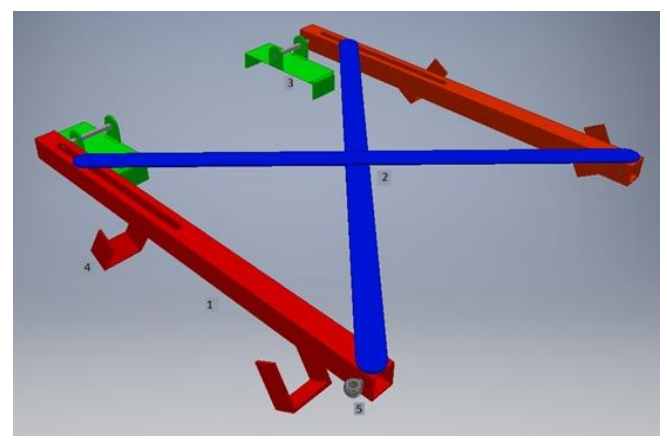

Fig 10: unfolded Solution



Fig 11: folded Solution

\section{Conclusion}

It is possible to adapt a self-balancing scooter to a conventional wheelchair with a simple mechanism. Resulting in an economic motorized wheelchair which can be manufactured over a low-cost parameter. All used materials like the self-balancing scooter, wood and metal can be purchased in any electrical- and hardware store except for the steering system, which resembles a bike shifting system. Based on the function of this solution the main wheels of the wheelchair have to be lifted off the ground. This proposed construction is 
kept simple and could be rebuilt by non-professional craftsman at home. This increases the accessibility tremendously and contributes to the satisfaction of the high demand of motorized wheelchairs.

Regarding the aeging of population, the solution is a potential contribution for the increasing needs of assisted mobility. The aim of the work is to contribute with a proposal which is openly accessible via the internet for the end user. So they can copy and build this mechanically simple construction.

However, the proposal is done with room for improvement. Those improvements could be a possible external interface, reducing the mechanical complexity, an extra batteries or a charging device that is directly accessible from the wheelchair.

\section{Acknowledgements}

First and foremost, we would like to express our deep and sincere gratitude to our research supervisor Guillermo Peris-Fajarnés as well as Bernardo Pajares Moreno, María MonchoSantonja and Ismael Lengua for their support throughout our work. We would also like to give a huge thank you to Professor Pedro Fuentes by giving us his support and providing invaluable guidance throughout our project. Finally, a major thanks you to the School of Engineering and Design, the Research Centre in Graphic Technologies and the Universidad Politècnica de Valencia for providing us with a workspace and allowing us to use their workshops.

\section{References}

AidWheels Mobility Solutions. (2019). Retrieved from https://aidwheels.com/home/

Analysis of Wheelchair Need. (2019). Retrieved from https://www.wheelchairfoundation.org/programs/from-the-heart-schools-program/materials-andsupplies/analysis-of-wheelchair-need/

Hollingshead, T. (2015). BYU students make the world's lightest, least expensive motorized wheelchair. BYU News. Retrieved from https://news.byu.edu/news/byu-students-make-worldslightest-least-expensive-motorized-wheelchair

Hudakova, A., \& Hornakova, A. (2011). Mobility and quality of life in elderly and geriatric patients. International Journal Of Nursing And Midwifery, 3(7), 81-85. Retrieved from http://www.academicjournals.org/app/webroot/article/article1379411601_Hudakova\%20and\%20 $\% 20$ Hornakova.pdf 
Patent CN204223088U (2015). Accepted on March 25, 2015 in China. Retrieved from Google Patents Public Datasets

United Nations Department of Economic and Social Affairs. (2017). World Population Ageing (pp. 17). United Nations.

Wheelchair Foundation. (2019). Retrieved from https://www.wheelchairfoundation.org

WHO. (2015). World report on ageing and health (p. 182). Luxembourg: World Health Organisation.

Winter, A. (2012). The cheap all-terrain wheelchair. Speech, Boston. 